\title{
Working towards clinical effectiveness-a multi-disciplinary approach to robotic surgery
}

\author{
Anthony M. Garbutt \\ Department of Nursing, Midwifery and Health, Northumbria University, Coach Lane Campus, Newcastle-Upon-Tyne, UK \\ Correspondence to: Anthony M. Garbutt. Lecturer in Adult Nursing, Department of Nursing, Midwifery and Health, Northumbria University, Coach \\ Lane Campus, Newcastle-Upon-Tyne, UK. Email: Anthony.m.garbutt@northumbria.ac.uk.
}

\begin{abstract}
The importance of a multi-disciplinary team (MDT) approach to conventional surgical techniques has strong empirical support. The MDT approach to robotic-assisted surgery (RAS) has not been clearly defined, which encourages prospectively poor MDT performance. Poor performance of the MDT approach, allied to the constant evolution of technology-assisted surgery, can generate unacceptable operative and patient outcomes. This review offers a nursing perspective to the complex paradigm of thoracic RAS, demonstrating key indicators to perioperative MDT engagement. This will be achieved by offering a rationale for RAS in pulmonary resection, identifying additional surgeries where utility is demonstrated. Evaluation of the available evidence will synthesize clinical quality indicators, while key strategies in effective MDT development can be summarised. Conclusively, bespoke and experiential knowledge will be shared, based upon the investigatory findings discussed throughout this article. Allied to a recommended developmental framework, this perspective should allow for transfer of knowledge, creation and replication of useful interventions. Lung cancer is an ever-increasing global concern, currently being the co-modal cancer with an estimated 2.09 million cases worldwide. Populations are ageing and with annual global costs of at least \$1.16 trillion, effective treatments are required. RAS shows promise in treating large and complex lesions when compared to a video-assisted thoracoscopic surgery (VATS) approach. A critical indicator being enhanced vision and dexterity in comparison to a VATS approach. Economically, RAS has proven to be an expensive technique, however, when initial purchase costs are excluded, intra-operatively, there are ways to narrow the expense gap and make RAS cheaper. When assessing per hospital stay, exclusive of initial purchase cost, RAS is found to be cheaper than open thoracotomy. This article demonstrates that RAS for pulmonary resection has utility for complex lesions where a VATS approach would be unsuitable. Crucially, as with all complex surgery, the MDT must be performed effectively for optimum patient outcomes.
\end{abstract}

Keywords: Thoracic surgery; robotic-assisted surgery (RAS); assistive technology; multi-disciplinary teamwork; clinical effectiveness

Submitted Aug 30, 2018. Accepted for publication: Jan 23, 2019.

doi: 10.21037/acs.2019.02.01

View this article at: http://dx.doi.org/10.21037/acs.2019.02.01

\section{Introduction}

The World Health Organisation (1) has identified lung cancer as the co-modal cancer worldwide, alongside breast cancer. There are currently 2.09 million reported cases of lung cancer worldwide, which led to 1.76 million deaths over a one-year period, with an estimated $80 \%$ of these cancers caused through tobacco smoking or exposure (1).

Seminally, Doll \& Hill (2) identified a link between smoking and lung carcinoma, however, noted $31.7 \%$ of their sample were non-smokers with lung carcinoma. Cufari et al. (3) more recently discussed a trend in 'never-smokers' with lung cancer, identifying a $15 \%$ increase in lung cancer rates of non-smokers between 2008 and 2014. Interestingly, Cufari et al. (3) concluded there is little evidence to attribute the rise to any single cause. Tomasetti \& Vogelstein (4) tentatively suggest 'replicative mutations' in stem cell 
division could be a relatively unexplored causation in cancer, with the risk of development increasing with age.

Prospectively, the Global Burden of Disease Cancer Collaboration (5) note a 29\% rise in tracheal, bronchus and lung (TBL) cancers since 2005; 18\% attributable to ageing population and $12 \%$ to population growth. In the UK, the Government Office for Science (6) suggest a national population growth of 9.7 million people from 2014 to 2039 (from 64.6 to 74.3 million). The highest percentage growth will be in the over 60 age group, prospectively increasing from 14.9 to 21.9 million. This indicates over $70 \%$ of the population increase will be in the over 60 age group. This may potentially increase the number of cancer diagnoses due to replicative mutation.

When assessing the costs of cancer globally, the 2010 figure was $\$ 1.16$ trillion (7). This will have elevated when allied with an increase in the ageing population (6). Lifestyle risk factors in cancer have increased in propensity and are identified as: increased alcohol intake, unhealthy diet and physical inactivity (1). Screening and early diagnosis are key in reducing the burden of cancer on a healthcare organisation; while adequate pathology systems will improve the chances of good outcomes (1). Effective cancer screening, early diagnosis and pathological sampling will often lead to surgery, requiring a highly specialised multidisciplinary team (MDT).

A growth area within UK health and social care is assistive health technology, and due to the rapid evolution of this sector, it is difficult to accurately judge the scale of their impact (6). One assistive technology currently used in global healthcare is the Intuitive Surgical DaVinci robotic system, in Si and concurrently $\mathrm{Xi}$ and $\mathrm{X}$ platforms (Intuitive Surgical Inc.). Robotic-assisted surgery (RAS) for pulmonary resection has been described in literature since the early 2000's. RAS is shown to have utility for anterior mediastinal surgery (Myasthenia Gravis and Thymectomy), posterior mediastinal tumours (neurogenic tumours, lymphatic system tumours and oesophageal cyst removal), oesophageal resection and Heller's Myotomy (8).

\section{Evidence in thoracic RAS}

The most common surgical robotic system is the Intuitive Surgical Inc., DaVinci system. This comes in Si and concurrently $\mathrm{Xi}$ and $\mathrm{X}$ forms (Intuitive Surgical Inc.) (9). This system offers provision for a maximum of 4 robotic arms to be operated remotely by an adequately trained surgeon $(3 \mathrm{~mm} \times 8 \mathrm{~mm}$ instrumentation arms and $1 \mathrm{~mm} \times$
$12 \mathrm{~mm}$ camera arm).

Seminally, Melfi et al. (10) described their early experience of RAS in pulmonary resection. Five patients underwent RAS, with all 5 having peripheral opacities (three stage 1 bronchogenic carcinoma and two typical carcinoid lesions). Of the 5 patients, 2 were converted to mini-thoracotomy with no technical mishap cited. It was concluded that RAS pulmonary resection is possible, however, they noted that considerable improvements were needed for safe and expeditiously performed pulmonary lobectomy.

Louie et al. (11) illustrated their comparative early experience of 40 RAS lobectomies versus 35 mature technique video-assisted thoracoscopic surgery (VATS) lobectomies. They illustrated their results of RAS versus VATS as follows: Mean operative time (213 versus 208 minutes), blood loss (153 versus $134 \mathrm{~mL}$ ), intensive care stay ( 0.9 versus 0.6 days) and length of stay ( 4.0 versus 4.5 days). They indicated similar results between techniques, concluding RAS to be at no technical disadvantage.

Expanding sample sizes, Dylewski et al. [2011] identified 200 consecutive RAS pulmonary resection cases (stating 154 lobectomy, 4 bilobectomy, 35 segmentectomy, 3 sleeve lobectomy, 3 en bloc resection and 1 pneumonectomy). Median values were: 90 -minute operating time, $70 \mathrm{~mL}$ intraoperative blood loss and 3-day hospital stay. Sixtyday mortality and morbidity rates were $2 \%$ and $26 \%$ respectively, with the largest lesion at $8.5 \mathrm{~cm}$. VATS limitations are cited as counterintuitive orientation, 2-dimensional imaging, reduced depth perception and limited instrument manoeuvrability. RAS is suggested to be technically superior in these areas.

Dylewski et al. (12) identified patient selection criteria for RAS as adequate cardiopulmonary reserve, peripherally located lesion(s) and pathologically proven non-small cell lung carcinoma (NSCLC), no clinical or pathological evidence of mediastinal nodal involvement and no lesion located in close proximity to hilar structures. Cerfolio et al. [2011a] supportively identified NSCLC, adequate cardiopulmonary reserve and mediastinal lymph node negative as RAS selection criteria, and additionally, advocated for pre-operative computerised tomography (CT) scan, positron emission tomography (PET), stress test and pulmonary function (within normal reference ranges). Interestingly, Cerfolio et al. [2011b] stipulated RAS exclusion criteria as segmental bronchus or more proximal tumour location, chest wall involvement requiring rib resection, N2 node positive and patient refusal of RAS. 
When consent is received for RAS, both Dylewski et al. (12) and Cerfolio et al. (13) conclude that potentially superior lymphadenectomy is possible over VATS and open surgery. Cerfolio et al. (13) identified a consecutive sample of 150 patients, concluding all cancer patients received a margin negative (R0) resection and all visible N1 and N2 lymph nodes can be completely extirpated, citing a median of 14 lymph nodes removed for NSCLC.

Cerfolio et al. (14) identified a consecutive patient sample of 168, with 148 undergoing RAS surgery (7 with metastatic pleural disease and 13 conversions, with 2 due to intraoperative bleeding). All cancer patients underwent R0 resection, with a median of five N2 and three N1 nodal stations, concluding RAS technique for pulmonary resection is "oncologically sound". Cerfolio et al. (14) compellingly compared RAS pulmonary resection to propensity-matched nerve-sparing thoracotomy. One hundred forty eight patients underwent RAS (106 lobectomy cases, 26 wedge resection and 16 segmentectomy) versus 318 nerve-sparing thoracotomy cases. A provocative case was presented for VATS being an unsuitable comparison to RAS due to limitations in lesion ability in VATS (lesion size $<4 \mathrm{~cm}$, without N1 disease). The largest RAS resection in this study was $9.4 \mathrm{~cm}$, potentially indicating a superior level of dexterity in RAS over VATS.

Cerfolio et al. (14) described clinical results of nervesparing thoracotomy versus RAS are consecutively: morbidity $38 \%$ versus $27 \%$, mortality $3.1 \%$ versus $0 \%$, chest drain duration 3.0 days versus 1.5 days, post-operative pain score median and range at 3 weeks $4.4[0-8]$ versus 2.5 $[0-7]$ and hospital stay median and range 4.0 [1-67] versus 2.0 [1-7]. Interestingly, post-operative RAS morbidities are identified as transient atrial fibrillation (12 patients), air leak (10 patients), chylothorax (2 patients), pneumothorax post drain removal (2 patients), subcutaneous emphysema (6 patients) and respiratory distress requiring reintubation (1 patient).

Cerfolio et al. (13) illustrated a completely portal robotic lobectomy with 4-arm (CPRL-4), building upon the work on Ninan \& Dylewski (15) who described a totally portal 3-arm approach without utility thoracotomy as technically possible. Cerfolio and colleagues (13) indicated the main benefit of CPRL-4 over 3-arm is autonomous lung retraction, also streamlining specimen extraction. Secondary benefits include 3-dimensional vision to improve precision. Crucially, full team training is identified as critical to optimise patient safety.
RAS can be an expensive technique. A US study by Park \& Flores (16) suggests costs up to $\$ 3,981$ more than VATS, however, it can potentially be $\$ 3,988$ less than open thoracotomy. A more contemporary cost-analysis came from a sample of 112 patients in France (17), identifying 57 RAS versus 55 VATS pulmonary resections, with median operative costings of $€ 13,424$ versus $€ 11,759$ respectively. Additionally, you must also factor in initial purchase costs. In the UK, they are roughly $£ 1.7$ million, with maintenance costs of an estimated $£ 10,000$ per annum $(18,19)$.

In a large-scale comparative study of 15,502 lung resection patients from 305 hospitals (665 underwent RAS, 335 lobectomies, 330 wedge resection). Swanson et al. (20) identified total hospital costs for RAS and VATS lobectomy as $\$ 25,040.70$ versus $\$ 20,476.60$ respectively; with wedge resection totalling $\$ 19,592.40$ versus $\$ 16,600.10$ respectively. Incidentally, Gondé et al. (17) had found similar findings, however, noted the open conversion rate of VATS and RAS as $16 \%$ and $2 \%$ respectively, potentially suggesting a more dextrous, therefore safer approach in RAS. This is congruent to the suggestion of meticulous and relatively haemostatic dissections in RAS (12).

\section{Barriers to effective RAS and rationale to the approach}

Jones \& Sethia (21) describe an increase in cost, elongated training periods and lack of strong contextual evidence as barriers to effective thoracic RAS. Antoniou et al. (19) additionally identify increased set-up times in RAS compared to VATS with difficulty in port docking as a clinical concern. RAS patients can experience increased anaesthetic times, potentially extending the patient recovery period and delaying hospital discharge (22). However, effective RAS team identification and engagement with shared vision will reduce operative times $(23,24)$.

RAS is criticised for lack of tactility and feedback for the surgeon compared to a VATS approach (25). Reduced tactility can be offset against increased dexterity, restoration of normal hand eye co-ordination and improved 3 -dimensional vision in RAS $(12,26)$. Tentative evidence can be found through comparative RAS versus VATS conversion to open rates- $16 \%$ versus $2 \%$ (17). Intriguingly, Lanfranco et al. (26), implies that RAS is awaiting trust in surgical technology to develop-identifying availability of 'telepresence surgery' as a low uptake approach due to potential technological distrust. 
Contextually, Ara Darzi, the foremost health minister in the UK, in his report 'Saws and Scalpels to Lasers and Robots', spoke favourably of keyhole surgery (27). He associated potentially reduced infection rates and reduced in-patient duration. He did, however, cite an underuse of RAS within the NHS. It was identified that RAS needed to prove its credentials against other minimally invasive techniques.

Interestingly, Brooks (28) suggests RAS is confounded by continually changing international evidence, promoting conceptualisation of bespoke empirical evidence to underpin interventions. Due to RAS having less suggested practical limitations in comparison to other minimal incision approaches $(12,14)$, it can be concluded that continuous collection, synthesis and publication of RAS data, specific to nation, or even region, could strengthen the contemporary evidence base.

\section{Team strategies to improve effectiveness}

Although you must be aware of cost implications and empirical evidence for commissioning RAS, initial drivers are cited as: passion for innovation, developing potential benefits and setting realistic goals or targets (29). Adopting these criteria for MDT selection is useful. Additionally, informing potential stakeholders of clinical benefits, as per prior evidence, will support MDT engagement (30).

Sharing goals will influence collaboration and decision making among the RAS MDT, identifying transferrable training and adequate skill mix amongst candidates (31). Particular attention should be paid to current Commissioning Policies Groups (CPGs) (if available) and literature review where appropriate to develop MDT competencies (32).

Effective teamwork in an operating theatre will include cooperation, leadership, coordination, awareness and communication (33). These factors can be reinforced using a robust framework. WHO (34) Surgical Safety Checklist (SSC) should be used. In a multi-national and economically diverse cohort of 3,955 patients, surgical mortality figures (within 30 days post-op) reduced from $1.5 \%$ to $0.8 \%$, with in-hospital complications reducing from $11 \%$ to $7 \%$ (35). Communication among surgeons, anaesthetists and nurses allied to the SSC is shown to improve standardisation of techniques, reducing risk of infection and avoidable complications (36). Hull et al. (37) found higher levels of MDT feedback improved technical surgical skills. Effective MDT communication will identify substantive deficiencies, reduce minor problems, leading to shorter, smoother and safer surgery (38). The WHO SSC allows for both pre-operative MDT communication and post-operative feedback through the debrief, encouraging essential iterative development.

Hollnagel et al. (39) offer a succinct recommendation of contemporary philosophical culture changes in healthcare. Moving from the notion of removing incidents altogether or 'as few things as possible go wrong' culture to accepting human fallibility, advocating an approach ensuring 'as many things as possible go right' - termed a safety-II approach. Braithwaite et al. (40) in a systematic review of 63 multinational articles concluded a positive workplace culture is significantly associated with system-related patient outcomes. System-related outcomes in healthcare are identified as: morbidity, failure to rescue, readmission rates and crucially, patient satisfaction. The importance of non-punitive feedback cannot be overstated to ensure safe, effective RAS.

Incident reporting will provide key performance feedback for improvements in healthcare (41). In the UK, Sujan et al. (42) identifies incident reporting as a key foundation of safe care, however, warns number of reported incidents will not translate instantaneously to clinical improvements. Part of a safety-II approach is learning from experience, encouraged through a positive culture (39). Part of learning from experience includes the importance of near miss reporting, which works informally through the WHO SSC. However, this must not be centralized, all clinical levels must receive incident feedback to ensure reduction in performance variability (42).

\section{Bespoke and experientially effective interventions}

The Human Factors in Healthcare document (43) advocates the enhancement of clinical performance through a development framework based upon teamwork, tasks, equipment, workspace, culture and organisation. This is applied to RAS below.

\section{Teamwork}

* Consistent RAS MDT for identified learning curve to improve standardisation of approach;

* Entire RAS team familiar with VATS and open thoracotomy;

* Identify nurses with experience in first-assistant role if available; 


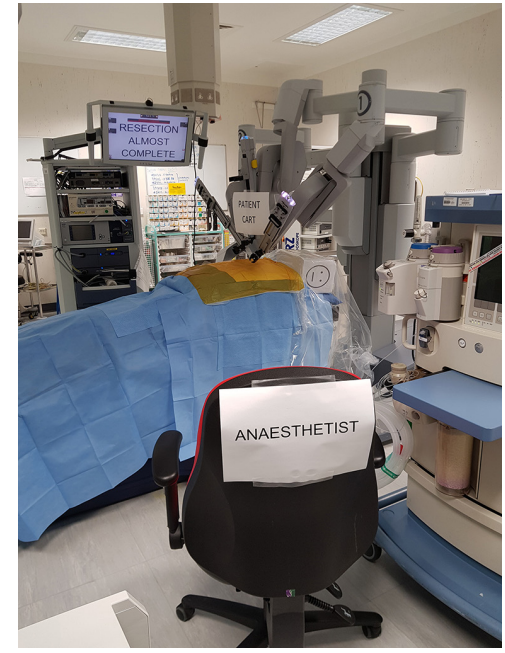

Figure 1 Anesthetist view (right sided surgery).

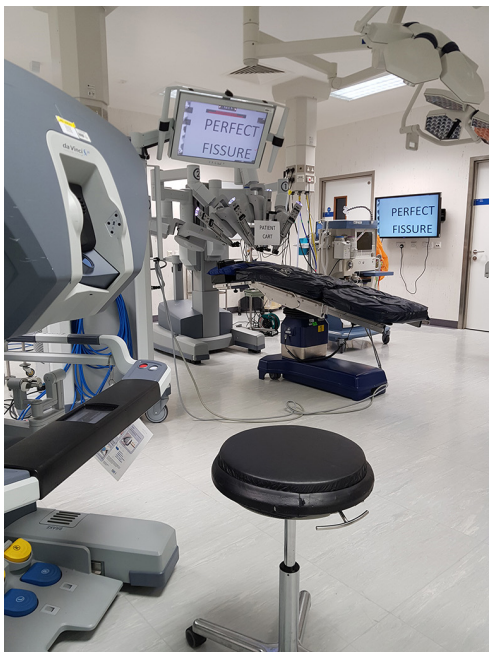

Figure 2 Surgeon's eye view.

- Anaesthetist has ready access to airway, including transparent drape (Figure 1);

* WHO SSC as feedback framework:

- Team HUG prior to anaesthetic induction (ideally around patient CT scan and/or X-ray);

- Set-up time, knife-to-skin, docking, un-docking, skin closure times and blood loss recorded;

- Full MDT SSC debrief performed.

Task

* Surgeon to discuss with MDT why RAS approach

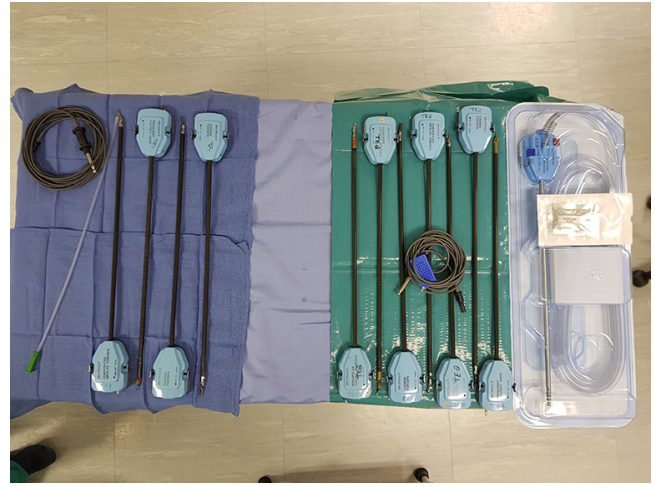

Figure 3 Reduced robotic instrumentation.

chosen;

* Patient operative positioning is paramount to effective RAS, aim for reproducibility (Figure 2);

* Proximity and ability for surgeon to see patient also important (Figure 2);

* Pre-decided conversion to open criteria-explicit plan B;

* Rigorous maintenance of binocular vision for surgeon;

* Identify time limit for RAS to avoid prolonged anaesthesia/team frustration

* Video recording of cases for review (if possible).

\section{Equipment}

* Cost reduction through reduced robotic instrumentation (Figure 3). $2 \times$ Cadiere Forceps, fenestrated Bipolar Forceps and Permanent Cautery Hook;

* Transparent drape for airway monitoring is essential (Figure 1);

* Non-linting, $10 \mathrm{~cm} \times 10 \mathrm{~cm}$ hydrophilic neurosurgical patties advisable (can be dried and re-used in situ to reduce $\mathrm{CO}_{2}$ loss);

* Blue coloured silastic vessel loops for contrast against background (consistently cut to $10 \mathrm{~cm}$ );

* Continuous evaluation of cost, effectiveness and alternative equipment;

\section{Workspace}

* Identify the largest operating room possible;

* Use the same operating room consistently-identify standardised landmarks for standardisation;

* Ensure additional slave monitors for improved MDT engagement; 
* Ensure surgeon has accessible view of patient and bedside assistant for effective communication (Figure 2).

\section{Culture/organisation}

* Engage with safety framework-WHO SSC;

* Debrief as an MDT for innovation (i.e., sub-xiphoid approach);

* Report incidents and near miss events;

* Encourage feedback to inform clinical standards;

* Advocate cross fertilisation among specialities;

* Use each case as a group learning exercise.

\section{Conclusions}

RAS is an effective approach to pulmonary resection, however, without a highly effective MDT, RAS will fail to deliver optimal patient benefits. As the co-modal global cancer, with over 2 million people currently suffering, there is a worldwide demand for highly effective treatment. Causation is widely acknowledged, with appropriate screening and early diagnosis available among many national healthcare systems. Surgery will become an option for many of the 2 million plus lung cancer sufferers.

Provocatively, VATS may be an unsuitable comparison for RAS due to counterintuitive orientation, 2-dimensional vision, reduced depth perception, inferior instrument manoeuvrability and utility for larger, more complex lesions in RAS.

Empirical evidence suggests RAS resection margins are reliable. Lymphadenectomy is suggested to be superior to VATS, however, not explicitly discussed in relation to open thoracotomy. Conversion rates in RAS (2\%) are as less than VATS (16\%), potentially due to improved dexterity and precision. Favourable morbidity and mortality rates are disclosed for RAS when compared to both VATS and open thoracotomy. Initial purchase costs are high for RAS at $£ 1.7$ million, with high annual maintenance at $£ 100,000$. RAS costings are $\$ 3-4,500$ higher per hospital stay than VATS, however, they are thought to be $\$ 4,000$ less than open thoracotomy.

RAS shows ability in complex thoracic surgery. As with any complex surgery, proposed ability is inconsequential without a highly performing team. The effectiveness of any thoracic RAS programme relies almost entirely on an MDT working towards an aspired outcome: shared team goals, passionate innovation and critical evaluation-more people than ever are relying on us to get it right.

\section{Acknowledgements}

None.

\section{Footnote}

Conflicts of Interest: The author has no conflicts of interest to declare.

\section{References}

1. WHO (2018). Available online: http://www.who.int/newsroom/fact-sheets/detail/cancer

2. Doll R, Hill AB. Smoking and carcinoma of the lung; preliminary report. Br Med J 1950;2:739-48.

3. Cufari ME, Proli C, De Sousa P, et al. Increasing frequency of non-smoking lung cancer: Presentation of patients with early disease to a tertiary institution in the UK. Eur J Cancer 2017;84:55-9.

4. Tomasetti C, Vogelstein B. Cancer risk: role of environment-response. Science 2015;347:729-31.

5. Global Burden of Disease Cancer Collaboration, Fitzmaurice C, Allen C, et al. Global, regional, and national cancer incidence, mortality, years of life lost, years lived with disability, and disability-adjusted life-years for 32 cancer groups, 1990 to 2015: a systematic analysis for the Global Burden of Disease Study. JAMA Oncol 2017;3:524-48.

6. Government Office for Science (2016) Future of An Ageing Population. Available online: https://www.gov.uk/ government/publications/future-of-an-ageing-population

7. WHO World Cancer Report (2014). Available online: http://publications.iarc.fr/Non-Series-Publications/ World-Cancer-Reports/World-Cancer-Report-2014

8. Kumar A, Asaf BB. Robotic thoracic surgery: The state of the art. J Minim Access Surg 2015;11:60-7.

9. Abrishami P, Boer A, Horstman K. Understanding the adoption dynamics of medical innovations: affordances of the da Vinci robot in the Netherlands. Soc Sci Med 2014;117:125-33.

10. Melfi FM, Menconi GF, Mariani AM, et al. Early experience with robotic technology for thoracoscopic surgery. Eur J Cardiothorac Surg 2002;21:864-8.

11. Louie BE, Farivar AS, Aye RW, et al. Early experience with robotic lung resection results in similar operative outcomes and morbidity when compared with matched video-assisted thoracoscopic surgery cases. Ann Thorac Surg 2012;93:1598-604; discussion 1604-5. 
12. Dylewski MR, Ohaeto AC, Pereira JF. Pulmonary resection using a total endoscopic robotic video-assisted approach. Semin Thorac Cardiovasc Surg 2011;23:36-42.

13. Cerfolio RJ, Bryant AS, Minnich DJ. Starting a robotic program in general thoracic surgery: why, how, and lessons learned. Ann Thorac Surg 2011;91:1729-36; discussion 1736-7.

14. Cerfolio RJ, Bryant AS, Skylizard L, et al. Initial consecutive experience of completely portal robotic pulmonary resection with 4 arms. J Thorac Cardiovasc Surg 2011;142:740-6.

15. Ninan M, Dylewski MR. Total port-access robot-assisted pulmonary lobectomy without utility thoracotomy. Eur J Cardiothorac Surg 2010;38:231-2.

16. Park BJ, Flores RM. Cost comparison of robotic, videoassisted thoracic surgery and thoracotomy approaches to pulmonary lobectomy. Thorac Surg Clin 2008;18:297300, vii.

17. Gondé H, Laurent M, Gillibert A, et al. The affordability of minimally invasive procedures in major lung resection: a prospective study. Interact Cardiovasc Thorac Surg 2017;25:469-75.

18. Alasari S, Min BS. Robotic colorectal surgery: a systematic review. ISRN Surg 2012;2012:293894.

19. Antoniou SA, Antoniou GA, Koch OO, et al. Robotassisted laparoscopic surgery of the colon and rectum. Surg Endosc 2012;26:1-11.

20. Swanson SJ, Miller DL, McKenna RJ Jr, et al. Comparing robot-assisted thoracic surgical lobectomy with conventional video-assisted thoracic surgical lobectomy and wedge resection: results from a multihospital database (Premier). J Thorac Cardiovasc Surg 2014;147:929-37.

21. Jones A, Sethia K. Robotic surgery. Ann R Coll Surg Engl 2010;92:5-8.

22. Parra-Davila E, Ramamoorthy S. Lap colectomy and robotics for colon cancer. Surg Oncol Clin N Am 2013;22:143-51, vii.

23. Higuchi T, Gettman T. Robotic Instrumentation, Personnel and Operating Room Set-up. In: Li MS. editor. Atlas of Robotic Urologic Surgery. New York, NY: Humana Press, 2011:15-30.

24. Kwak JM, Kim SH, Kim J, et al. Robotic vs laparoscopic resection of rectal cancer: short-term outcomes of a casecontrol study. Dis Colon Rectum 2011;54:151-6.

25. Lim DR, Min BS, Kim MS, et al. Robotic versus laparoscopic anterior resection of sigmoid colon cancer: comparative study of long-term oncologic outcomes. Surg Endosc 2013;27:1379-85.
26. Lanfranco AR, Castellanos AE, Desai JP, et al. Robotic surgery: a current perspective. Ann Surg 2004;239:14-21.

27. Darzi A. Saws and Scalpels to Lasers and Robots Advances in Surgery. Department of Health, London: DH, 2007.

28. Brooks P. Robotic-Assisted Thoracic Surgery for EarlyStage Lung Cancer: A Review. AORN J 2015;102:40-9.

29. Pawson R. The science of evaluation: a realist manifesto. London: Sage, 2013.

30. Payne TN, Pitter MC. Robotic-assisted surgery for the community gynecologist: can it be adopted? Clin Obstet Gynecol 2011;54:391-411.

31. Randell R, Honey S, Hindmarsh J, et al. A realist process evaluation of robot-assisted surgery: integration into routine practice and impacts on communication, collaboration and decision-making. Southampton (UK): NIHR Journals Library, 2017 Jun. Health Services and Delivery Research.

32. Wiles LK, Hibbert PD, Stephens JH, et al. STANDING Collaboration: a study protocol for developing clinical standards. BMJ Open 2017;7:e014048.

33. Healey AN, Undre S, Vincent CA. Developing observational measures of performance in surgical teams. Qual Saf Health Care 2004;13:i33-40.

34. WHO (2008). Available online:: http://www.who.int/ patientsafety/safesurgery/ss_checklist/en/

35. Haynes AB, Weiser TG, Berry WR, et al. A surgical safety checklist to reduce morbidity and mortality in a global population. N Engl J Med 2009;360:491-9.

36. Pugel AE, Simianu VV, Flum DR, et al. Use of the surgical safety checklist to improve communication and reduce complications. J Infect Public Health 2015;8:219-25

37. Hull L, Arora S, Aggarwal R, et al. The impact of nontechnical skills on technical performance in surgery: a systematic review. J Am Coll Surg 2012;214:214-30.

38. Catchpole KR, Giddings AE, Wilkinson M, et al. Improving patient safety by identifying latent failures in successful operations. Surgery 2007;142:102-10.

39. Hollnagel E, Wears RL, Braithwaite J. From Safety-I to SafetyII: A white paper. Published simultaneously by the University of Southern Denmark, University of Florida, USA, and Macquarie University, Australia: The Resilient Health Care Net; 2015. Available online: http:// resilienthealthcare.net/onewebmedia/WhitePaperFinal.pdf

40. Braithwaite J, Herkes J, Ludlow K, et al. Association between organisational and workplace cultures, and patient outcomes: systematic review. BMJ Open 2017;7:e017708. 
41. Braithwaite J, Westbrook MT, Travaglia JF, et al. Cultural and associated enablers of, and barriers to, adverse incident reporting. Qual Saf Health Care 2010;19:229-33.

42. Sujan M, Huang H, Braithwaite J. Why do healthcare organisations struggle to learn from experience? A SafetyII perspective. Healthcare Systems Ergonomics and

Cite this article as: Garbutt AM. Working towards clinical effectiveness-a multi-disciplinary approach to robotic surgery. Ann Cardiothorac Surg 2019;8(2):255-262. doi: 10.21037/ acs.2019.02.01
Patient Safety Conference (HEPS 2016) 342-48. Available online: https://warwick.ac.uk/fac/sci/med/staff/sujan/ research/resilience/heps2016_sujan.pdf

43. NHS England (2013). Available online: https://www. england.nhs.uk/wp-content/uploads/2013/11/nqb-humfact-concord.pdf (accessed: 15th August 2018). 